\title{
3D Network exploration and visualisation for lifespan data
}

\author{
Rolf Hühne ${ }^{1,2 \dagger}$, Viktor Kessler ${ }^{1,3 \dagger}$, Axel Fürstberger ${ }^{1 \dagger}$, Silke Kühlwein ${ }^{1}$, Matthias Platzer², \\ Jürgen Sühnel ${ }^{2}$, Ludwig Lausser ${ }^{1}$ and Hans A. Kestler ${ }^{1,2^{*}}$ (D)
}

\begin{abstract}
Background: The Ageing Factor Database AgeFactDB contains a large number of lifespan observations for ageing-related factors like genes, chemical compounds, and other factors such as dietary restriction in different organisms. These data provide quantitative information on the effect of ageing factors from genetic interventions or manipulations of lifespan. Analysis strategies beyond common static database queries are highly desirable for the inspection of complex relationships between AgeFactDB data sets. 3D visualisation can be extremely valuable for advanced data exploration.

Results: Different types of networks and visualisation strategies are proposed, ranging from basic networks of individual ageing factors for a single species to complex multi-species networks. The augmentation of lifespan observation networks by annotation nodes, like gene ontology terms, is shown to facilitate and speed up data analysis. We developed a new Javascript 3D network viewer JANet that provides the proposed visualisation strategies and has a customised interface for AgeFactDB data. It enables the analysis of gene lists in combination with AgeFactDB data and the interactive visualisation of the results.

Conclusion: Interactive 3D network visualisation allows to supplement complex database queries by a visually guided exploration process. The JANet interface allows gaining deeper insights into lifespan data patterns not accessible by common database queries alone. These concepts can be utilised in many other research fields.
\end{abstract}

Keywords: Lifespan, Ageing, Gene network, 3D visualization, Ageing factor database, AgeFactDB, Differentially expressed genes

\section{Background}

In ageing research, the lifespan of an organism is an indicator for determining factors that play a role in this process. These ageing factors (AFs) can be genes, chemical compounds or other factors like dietary restriction. Usually, they are examined under different experimental conditions in model organisms like the worm (Caenorhabditis elegans), yeast (Saccharomyces cerevisiae), fruit fly (Drosophila melanogaster), mouse

\footnotetext{
*Correspondence: hans.kestler@uni-ulm.de

Ludwig Lausser and Hans A. Kestler are joint senior authors.

${ }^{\dagger}$ Rolf Hühne, Viktor Kessler and Axel Fürstberger contributed equally to this work.

'Institute of Medical Systems Biology - Ulm University, Albert-Einstein-Allee 11, 89081 Ulm, Germany

${ }^{2}$ Leibniz Institute on Aging - Fritz Lipmann Institute, Beutenbergstr. 11, 07745 Jena, Germany

Full list of author information is available at the end of the article
}

(Mus musculus), and many others. The results of these experiments may be extracted from the scientific literature in the form of lifespan observations (LOs). They describe the effect of interventions at AFs on the lifespan of the model organism.

In a lifespan experiment, a single AF or a combination of two or more AFs can be involved. The intervention can be different for each AF. For example, a knock-out of gene $A$ could be coupled to the overexpression of gene $B$. Also, AFs can be involved in different experiments together with different other AFs. For example, some genes like daf- 2 and daf- 16 from C. elegans were tested in several hundred AF combinations and various interventions, e.g. $[1,2]$. The effects on the lifespan of the organism may also differ drastically.

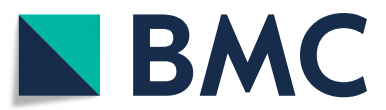

(c) The Author(s). 2018 Open Access This article is distributed under the terms of the Creative Commons Attribution 4.0 International License (http://creativecommons.org/licenses/by/4.0/), which permits unrestricted use, distribution, and reproduction in any medium, provided you give appropriate credit to the original author(s) and the source, provide a link to the Creative Commons license, and indicate if changes were made. The Creative Commons Public Domain Dedication waiver (http://creativecommons.org/publicdomain/zero/1.0/) applies to the data made available in this article, unless otherwise stated. 


\section{D Network visualisation}

Dealing with this heterogeneity and the complexity of the relationships between the LOs is a major challenge in gaining a comprehensive overview or in generating an integrative model. Visualisation techniques can aid in analysing this complex data [3-5] and also help to generate new hypotheses not only on a quantitative level [6].

In line with these supportive approaches on sets, network visualisations can assist in organising vast amounts of data according to known relationships or properties. $3 \mathrm{D}$ visualisation can help researchers on special occasions in this task, although 2D representations should generally be preferred [7]. In the lifespan network visualisation context we present here, 3D networks outperform their 2D counterparts regarding compactness and layout. While $3 \mathrm{D}$ embeddings allow a compact representation of hundreds or thousands of nodes, 2D embeddings result in a significant expansion, increasing navigation costs (see Additional file 1: Figure S1). Furthermore, it is known that any finite graph can be embedded into a threedimensional space such that no pair of edges crosses [8]. As LO networks cannot be guaranteed to be planar graphs, 2D embeddings might also result in intersecting edges, while in 3D these non-intersecting representations exist [8].

Additionally, psychophysical experiments provide evidence that the human primary visual processing system is specifically designed to process $3 \mathrm{D}$ information. Nakayma et al. indicate the parallel processing of attribute information like the colour from different depth planes [9]. Enns et al. give evidence that 3D objects with lightningrelated depth cues accelerate the visual search in comparison to 2D objects [10]. Xu et al. report an increased capacity of the visual short-term memory (VSTM) [11] when objects are distributed between different layers [12].

The benefits of 3D network representations come at the risk of visual occlusion and possible perspective distortion [13]. Due to the layered structure of 3D representations, elements in the foreground can mask elements in the background. Which can be overcome by interactive graph manipulation such as zooming, rotating, panning and filtering.

Perspective distortions might occur when object sizes and distances are modified for both data visualisation and perspective depth effects. They can be omitted by ignoring depth calculations. Similar risks, such as diminished legibility of text, can be avoided by excluding these objects from other perspective transformations (i.e. rotations).

Overall 3D visualisation has many advantageous unique selling points. Its disadvantageous can be mitigated via interactive graph exploration and manipulation.

\section{AgeFactDB}

The public JenAge Ageing Factor Database AgeFactDB [14] contains LOs for AFs. Table 1 provides an overview of the number of AFs and observations by type (lifespan, other ageing phenotypes, homology analysis) and by their ageing relevance evidence type (experimental, computational).

Currently, the core of AgeFactDB is a collection of about 2600 genes for which LOs and other experimental evidence were gathered from experiments with different model organisms (experimental AFs). This set was extended by about 14,000 genes gained in a homology analysis using data from the homology database HomoloGene [15] (putative AFs).

Overall, AgeFactDB contains about 9500 observations. About 1000 are free-text descriptions of ageing phenotypes, and about 7000 are structured LOs. Besides, there are about 1500 homology analysis observations, each resembling a homology group.

As an example for structured LOs, Table 2 shows observation OB_000094 [16] involving the genes FOB1, SIR2 and TOR1 from S. cerevisiae. The deletion of these three genes resulted in a $33.5 \%$ increase in lifespan. Note that there are two different types of lifespan defined for yeast: chronological lifespan and replicative lifespan [17]. The chronological lifespan is the number of days which a specific yeast cell is living. The replicative lifespan is a measure of the total number of daughter cells generated by a mother cell [18].

\section{D Network viewer}

Aside from commercial tools, there are a few other freely available 3D visualisation tools. NetworkX [19]

Table 1 AgeFactDB Statistics. Overview of the number of AFs and observations in AgeFactDB Release 1 by type and by their ageing relevance evidence type

\begin{tabular}{lllll}
\hline \multicolumn{5}{c}{ Ageing relevance evidence } \\
\cline { 2 - 5 } & Experimental & Computational & Both & Any \\
\hline Ageing factors & $\mathbf{2 7 4 3}$ & $\mathbf{1 4 4 3 7}$ & $\mathbf{5 8 1}$ & $\mathbf{1 6 5 9 9}$ \\
Genes & 2594 & 14437 & 581 & 16450 \\
Chemical & 91 & - & - & 91 \\
compounds & & & - & 58 \\
Other factors & 58 & - & - & $\mathbf{9 6 1 1}$ \\
Observations & $\mathbf{8 1 5 9}$ & $\mathbf{1 4 5 2}$ & - & 7219 \\
$\begin{array}{l}\text { Lifespan } \\
\text { (structured) }\end{array}$ & 7219 & - & & \\
$\begin{array}{l}\text { Ageing phenotype } \\
\text { (unstructured text) }\end{array}$ & 940 & - & - & 940 \\
Homology analysis & - & 1452 & - & 1452 \\
\hline
\end{tabular}

Computational evidence refers to homology analysis observations, each resembling a homology group from the HomoloGene database [15]. The boldfaced numbers are the sums of the follow up rows 
Table 2 Example Observations from AgeFactDB

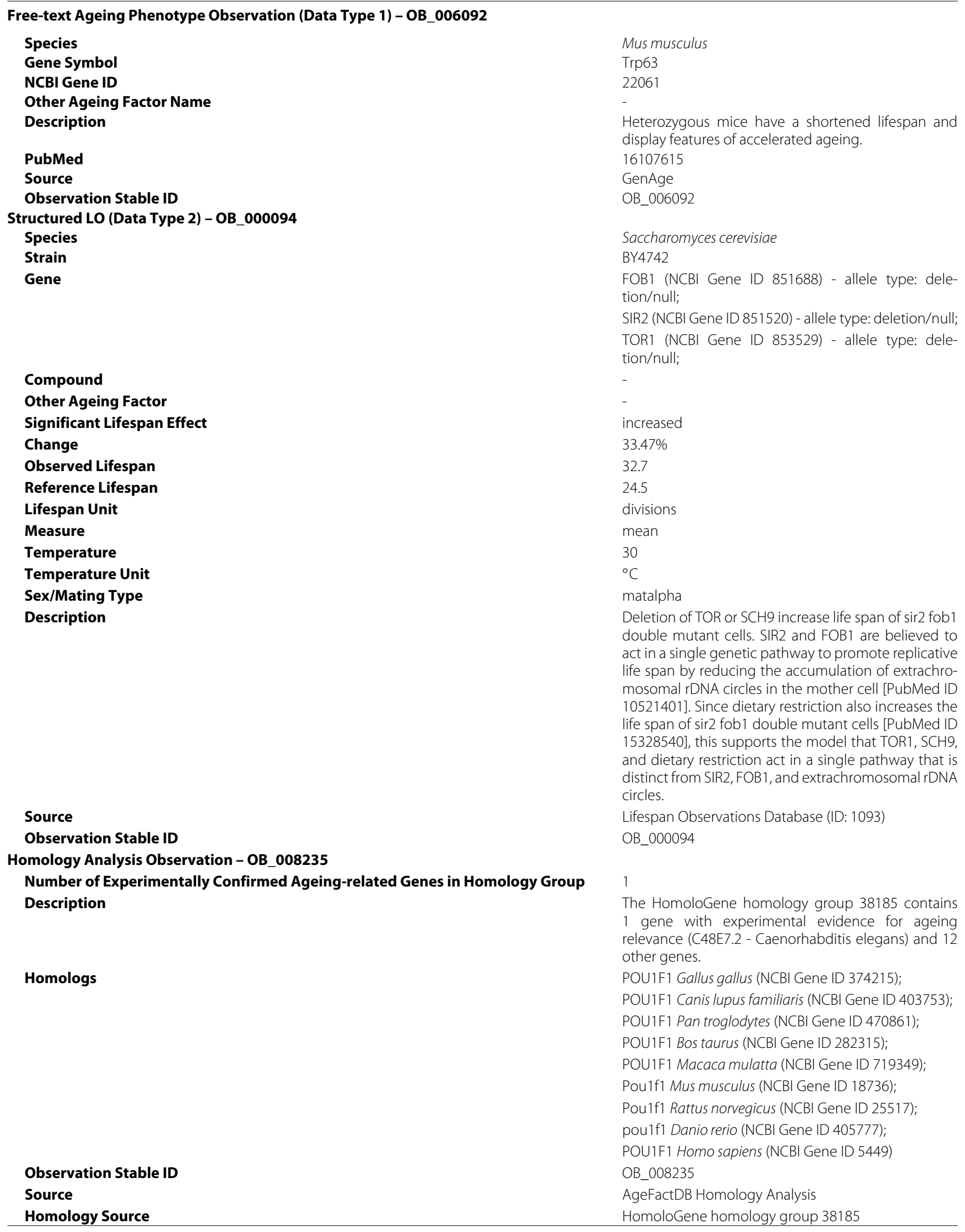


and iGraph [20] are examples for software packages that offer 3D network layout algorithms and the generation of static network images. The Javascript library vis.js provides its rudimentary viewer which has a user interface that enables only rotation, zoom, and translation of the network model [21]. Vanted [22] and SeeNet3D [23] are examples of specialised viewers for the domains of metabolic pathways and communication. The Cy3D plugin [24] for Cytoscape [25], a 2D network viewer in the biomedical domain, provides a $3 \mathrm{D}$ rendering engine. It is only suitable for small networks since it only supports rotation and zoom and no translation. BioLayout Express 3D [26] provides a functional layout algorithm for more extensive networks, the Fast Multipole Multilevel Method (FMMM) algorithm [27]. Nonetheless, the development of BioLayout Express 3D as a freely available tool ceased several years ago.

In the following, we present different types of networks and visualisation strategies for $\mathrm{LO}$ data. We show the benefit of augmentation of AF/LO networks by annotation nodes compared to AF annotation. Annotation nodes can be for example gene ontology (GO) [28] term nodes and KEGG pathway [29] nodes.

We also present a new Javascript network viewer with a customised interface for the visualisation of lifespan data from AgeFactDB, combined with user-provided genes of interest, for example, a list of genes differentially expressed during ageing. By two concrete example applications, we demonstrate the usefulness of the visualisation strategies and the network viewer.

\section{Methods}

JANet

JANet (Javascript AgeFactDB Network-viewer) is a specialised Javascript 3D network viewer for the visualisation of ageing-related network data from AgeFactDB. JANet extends the original design of the AgeFactDB by an interactive component allowing the user to browse the content of the database in a 3D graph representation. It facilitates the navigation through the data corpus of experimental evidence, citations and other background information via natural 3D movements and a well organised set of graph manipulations. JANet is also an interface allowing an untrained researcher to relate his data with the data corpus of AgeFactDB. By incorporating gene lists of interest in the original networks, JANet provides an embedding in the domain of ageing research.

As frontend, JANet provides a responsive HTML/ Javascript web browser interface, for an overview see Fig. 1. For the visualisation, it utilises the 3D Force-Directed Graph web component, based on ThreeJS/WebGL [30, 31] for 3D rendering and d3-force$3 \mathrm{~d}$ as the underlying physics engine for generating the

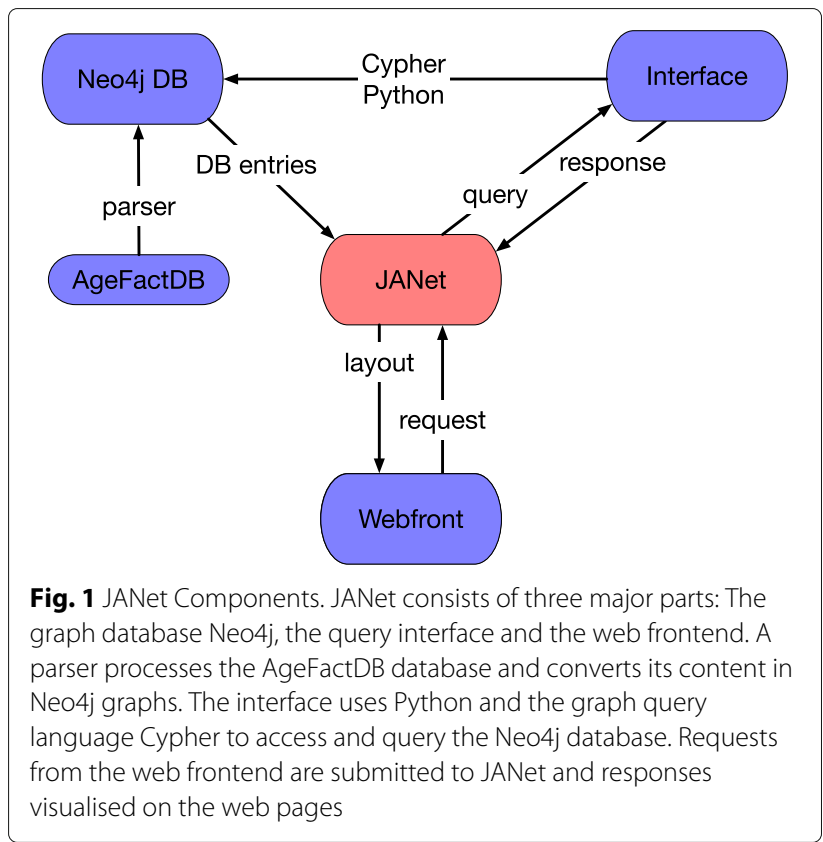

network layout. As the backend it has Python scripts and a Neo4j graph database [32].

\section{Interface}

The primary interface of JANet is structured into eight tabs which provide access to the main viewer for graph visualisation and the different options for network generation (Fig. 2a). Networks are generated according to three principles focusing on overviews of AFs, the inspection of individual AFs and the interaction of AFs with user-specified genes of interest. The interface additionally provides a statistical summary of the current database of AgeFactDB, help sides and the imprint. Generally, actions like node colouring and rendering work in the viewer on the currently selected nodes, enabling graph manipulations of node properties individually to create custom views.

\section{Viewer}

The Viewer tab provides the 3D graph representation of a chosen network and basic operations for graph editing (Fig. 2b). JANet comprises various options for customising the graph design and manipulating the graph layout. Among them, for example, changing the node colour and size. Network nodes can be marked with key information like the name or the lifespan change value. By hovering above a node with the mouse pointer, more detailed information about the node are given in the upper left corner of the viewer. The node itself is highlighted by a light blue halo. A white halo is shown when a node is selected. By selecting a region of interest (bounding box) sets of nodes can be selected or highlighted. The network can be 


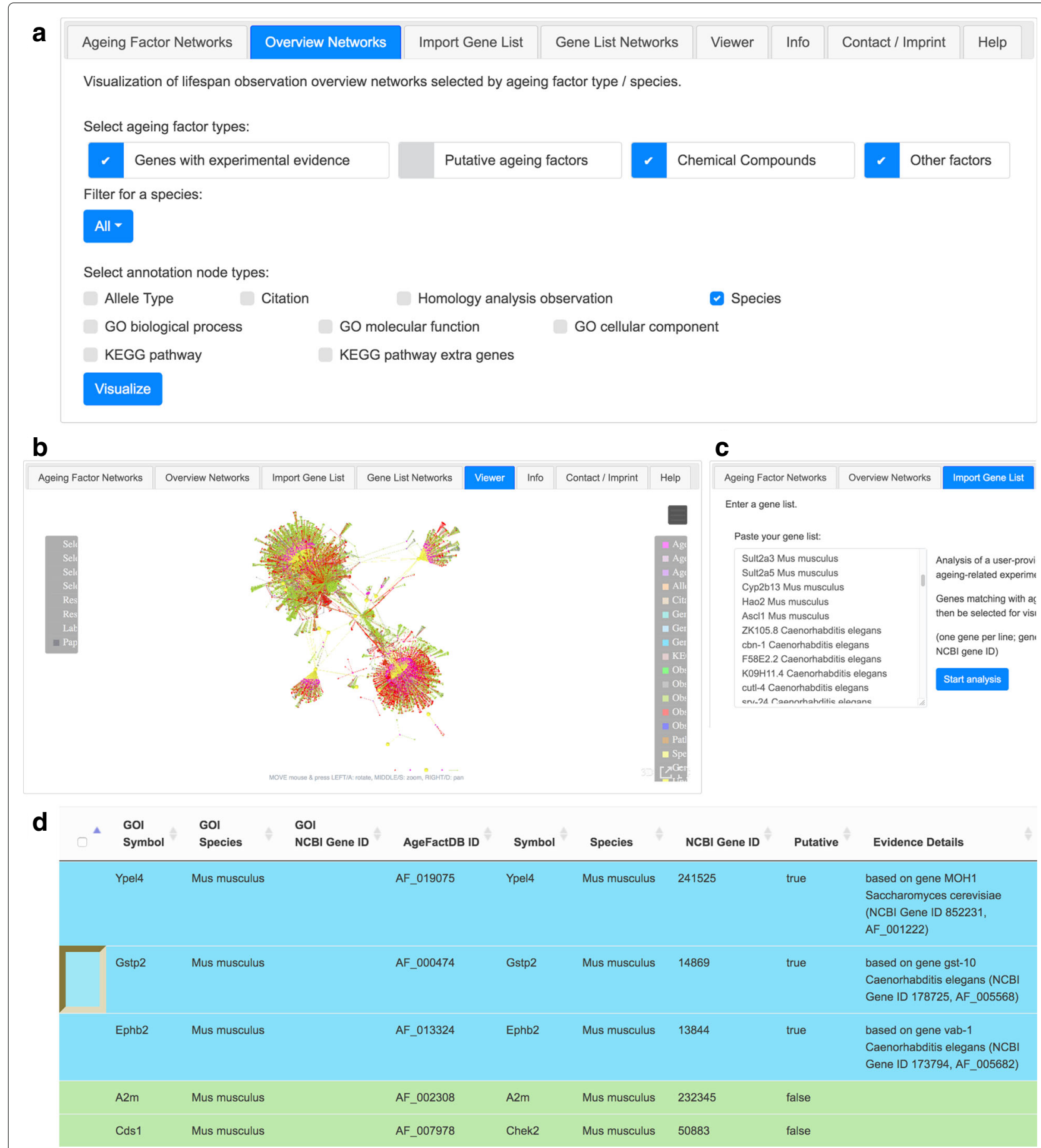

Fig. 2 JANet Graphical User Interface. a Screenshot of the JANet graphical user interface. The tab Overview Networks is selected. b The Viewer tab of JANet. The network containing all ageing factors (AFs) having lifespan observations (LOs), all LOs, and the corresponding species is shown. c The Import Gene List tab allows the user to provide his own gene list of interest. Those genes that are linked to AFs in AgeFactDB are afterwards listed in the Gene List Network tab. d The Gene List Network tab contains a list of the genes of interest that are known AFs or homologous AFs. It can be used to generate user specific networks

restricted to the selected node and its direct neighbours. The network viewer provides alternative view options. It can be expanded to a fullscreen mode or send to an independent browser tab/window. In this way, several networks can be analysed simultaneously. For a better 3D impression the viewer also offers a stereo view option. 


\section{Overview networks}

Rather holistic networks on all AFs of the AgeFactDB are generated in the tab Overview networks. The selection of AFs may be stratified according to the type of AF and the corresponding species. The tab also provides options for augmenting the graph with different kinds of annotation nodes. For example, they can provide additional information about the allele types or species. The constructed graph can afterwards be manipulated within the network viewer. Figure 2a gives an example of an overview network. It contains all AFs having LOs, all LOs, and the corresponding species.

\section{Ageing factor networks}

The networks generated by the tab Ageing Factor Networks are focused on one particular AF which is embedded either in its direct or complete neighbourhood. The functionality of this tab is comparable to the functionality of the tab for overview networks. Additionally, single AFs can be selected from a dropdown list.

\section{Gene list selection / Network}

JANet can be used as an interface for an integrative analysis combining the existing database of AgeFactDB and an user-specified list of genes of interest. Users can provide their list in the tab Import Gene List (Fig, 2c). A query to AgeFactDB returns a list of exact matches to ageing factors with experimental evidence and putative ageing factors. In the tab Gene List Network these results can be screened and edited (Fig. 2d). This tab also starts the network generation.

\section{Network layout algorithms}

Force layout algorithms position the nodes of a graph in two-dimensional or three-dimensional space so that all edges are of more or less equal length and as few crossing edges as possible are produced. Repulsive or attractive forces are assigned to edges and nodes based on their relative position. By minimising their energy the layout is generated step wise. Within JANet we utilise three different algorithms.

The 3d-forced-layout (3dFL) algorithm module implements a velocity Verlet numerical integrator for simulating physical forces on nodes. It is a numerical method used to integrate Newton's equations of motion [33]. In contrast the Fruchterman-Reingold (FR) algorithm focuses on even distribution of vertices, a minimal number of edge crossings and uniform edge length [34]. The fast multipole multilevel method (FMMM) is especially designed for separating substructures in large graphs [26].

\section{LO network visualisation techniques}

We use LO Networks as basic visualisation technique for the integration of different lifespan experiments. In these networks LOs and AFs are represented by nodes. Edges between both types of nodes link the AFs with the LOs in which they were involved. Multiple AFs can be connected to one LO and vice versa.

In order to facilitate the visual navigation, we utilise a colour code for the different node types. In the special case of LO nodes the colour indicates the direction of the lifespan change. An increased, decreased or unchanged lifespan is indicated by green, red or grey colour. The node size can be proportional to the quantitative lifespan change or other quantitative measures like the number of connections (degree). The edge colour is usually inherited from the node of a pair whose colour carries specific information for the other node. For AF/LO edges this is the LO node, whose colour usually indicates the direction of the lifespan change.

We present several visualisation techniques for LO networks (Fig. 3). In the "Results and discussion" section we present example networks, based on AgeFactDB data, to demonstrate the usage of these techniques.

\section{Direct neighbourhood}

For focusing on a specific AF only the direct neighbourhood of the AF is visualized (Fig. 3a). This includes the respective AF, all directly linked LOs, and all other AFs linked directly to these LOs.

This network type provides a compact view of the effects of all LOs in which an AF is involved directly.

In the network in Fig. $3 \mathrm{a}$ the $\mathrm{AF}$ in the focus is $A F_{1}$. $A F_{1}$ is linked to $3 \mathrm{LOs}\left(L O_{1}-L O_{3}\right) . A F_{2}$ is linked to $L O_{2}$ because it was tested together with $A F_{1}$ in the corresponding experiment. The same applies to $A F_{3}$ and $L_{3}$. No further AFs were tested in any of the 3 LOs.

In $L O_{1}$ the lifespan was decreased, indicated by the red node colour. In $\mathrm{LO}_{2}$ and $\mathrm{LO}_{3}$ the lifespan was increased, indicated by the green node colour.

\section{Complete neighbourhood}

The direct neighbourhood network is extended iteratively by including further AFs that were observed together with the neighbours of the AF in the focus (Fig. 3b). In this way the complete neighbourhood is included in the visualisation. The resulting network can be seen as the largest connected subgraph including the AF in the focus and all directly or indirectly connected LOs and AFs.

The complete neighbourhood network provides an overview on all experimentally analysed AF combinations and their effects on lifespan.

The direct neighbourhood network visualisation from Fig. 3a was expanded to the complete neighbourhood network visualisation in Fig. 3b. In the first expansion step $L O_{4}$ and $L O_{5}$ were added, linked to $A F_{2}$ but not to $A F_{1}$. Also $A F_{4}$ was added, linked to $L O_{5}$ together with $A F_{2}$. In the second expansion step $L O_{6}$ and $A F_{5}$, linked also to 


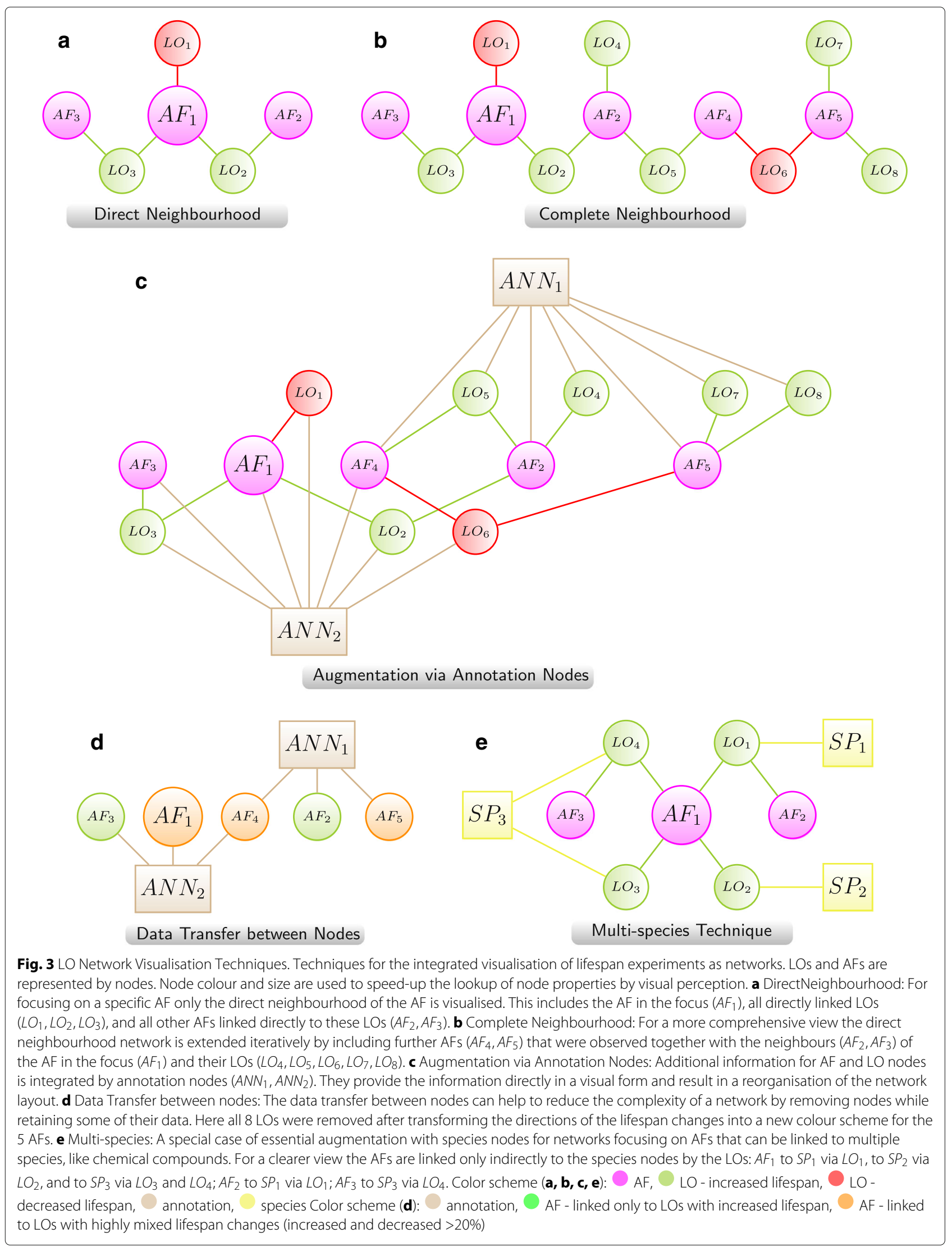


$L O_{6}$, were added. In the final expansion step $L O_{7}$ and $L O_{8}$ were added, both linked to $A F_{5}$.

\section{Augmentation via annotation nodes}

LO networks are augmented by additional information on either the AFs or the LOs or both. This is achieved by additional annotation nodes (ANNs). They provide the additional information directly in a visual form for all nodes at once. The augmented network visualisation in Fig. 3c shows an annotated version of the complete neighbourhood visualisation from Fig. 3b. The annotation node $A N N_{1}$ was linked to the $3 \mathrm{AFs}\left(A F_{2}, A F_{4}, A F_{5}\right)$ and to the $4 \mathrm{LOs}\left(\mathrm{LO}_{4}, \mathrm{LO}_{5}, L \mathrm{O}_{7}, \mathrm{LO}_{8}\right)$. The annotation node $A N N_{2}$ was linked to the $3 \mathrm{AFs}\left(A F_{1}, A F_{3}, A F_{4}\right)$ and to the $4 \mathrm{LOs}$ $\left(L O_{1}-L O_{3}, L O_{6}\right)$. This resulted in two groups around the annotation nodes $A N N_{1}$ and $A N N_{2}$, connected by the nodes $A F_{4}, \mathrm{LO}_{2}$, and $\mathrm{LO}_{6}$.

\section{Data transfer between nodes}

The data transfer between nodes is especially useful for reducing the complexity of a network by removing the nodes from which data was transferred. The transfer enables to retain some information from the removed nodes.

Figure $3 \mathrm{~d}$ shows a reduced version of the network from Fig. 3c. The collected qualitative lifespan change information from all LOs connected to an AF was transformed into a new colour for the AF. $A F_{2}$ and $A F_{3}$ are linked only to green $\mathrm{LO}$ nodes (indicating an increased lifespan) and got green as new colour. $A F_{1}, A F_{4}$, and $A F_{5}$ are linked to 2 or 3 green LO nodes and 1 red LO node (in the latter case indicating a decreased lifespan). These mixed effects were transformed into orange as new node colour.

The transferred data can also be used to define a new node size, which may be for example proportional to the maximal observed lifespan change. This information can already be helpful even if the LOs are not removed.

\section{Multi-species technique}

In contrast to genes, chemical compounds and other AFs can be linked to LOs of multiple species. This requires to augment such networks by species nodes (SP) that are linked to the corresponding LOs. We propose to leave out the links between the AFs and the species nodes. This will result in a much clearer and less complex network view, grouping the network clearly according to the involved organisms.

Because of the importance of the species information in these networks and the additional restriction to one type of species links we defined Multi-species as a separate technique.

The multi-species network visualisation in Fig. 3e contains $A F_{1}$ as multi-species AF. It is linked indirectly to all 3 species nodes $\left(S P_{1}-S P_{3}\right)$ by $4 \mathrm{LOs}\left(L O_{1}-L O_{4}\right)$. In $L O_{1}$ also $A F_{2}$ is involved, and in $L O_{2}$ also $A F_{3}$ is involved.
The layout shows 3 small groups centred around the multi-species node $A F_{1}$.

\section{Results and discussion}

After the basic description of the visualisation techniques and network types given in the "Methods" section, we first present concrete examples for some of the visualisation techniques introduced in the "Methods" section followed by use cases how these techniques were applied with JANet to solve specific tasks.

\section{LO network visualisation examples}

We show examples for the application to lifespan data for S. cerevisiae and C. elegans. All LOs were taken from AgeFactDB.

\section{Example 1: direct neighbourhood}

The direct neighbourhood example described here is focused on the AF TOR1 from S. cerevisiae (Fig. 4a). The gene belongs to the TOR signalling pathway, which has been shown to regulate lifespan across multiple species (S. cerevisiae, C. elegans, D. melanogaster, and M. musculus), as part of the TORC1 complex [35]. The direct neighbourhood consists of twenty LOs for TOR1 and five additional AFs (Dietary restriction, FOB1, GCN4, RPN4, SIR2) that are involved in these LOs.

The layout was calculated with the 3dFL algorithm. Different AF types are colour-coded: magenta indicates genes, dark purple indicates other factors, and light purple indicates chemical compounds (not present here). Each LO node is labelled with the lifespan change value, if available (n/a indicates a missing value).

Hovering over an LO node in the viewer provides additional information on the lifespan experiment. For this particular network all experiments were designed for the inactivation of TOR1. Those 15 LOs that are not connected to any other AF show a mean lifespan increase of $19 \%$ up to $56 \%$ for the inactivation of TOR1. In combination with the inactivation of the genes FOB1, GCN4, SIR2 are combined with dietary restriction, the lifespan was increased in the range of $19 \%$ up to $67 \%$. For an inactivation of the gene RPN4 and TOR1, a lifespan decline of $42 \%$ was observed.

By focusing on those LOs that involve TOR1 directly, the direct neighbourhood enables a quick overview of all 20 lifespan experiments involving it. The compact 3D view as a network can reveal the lifespan changes in combination with the other ageing factors more quickly than the large LO table for TOR1 available in AgeFactDB. Due to the rather small size with 26 nodes and 26 edges a $2 \mathrm{D}$ view is already helpful too.

\section{Example 2: complete neighbourhood}

The direct neighbourhood network of the AF TOR1 from the previous example was iteratively extended to the 


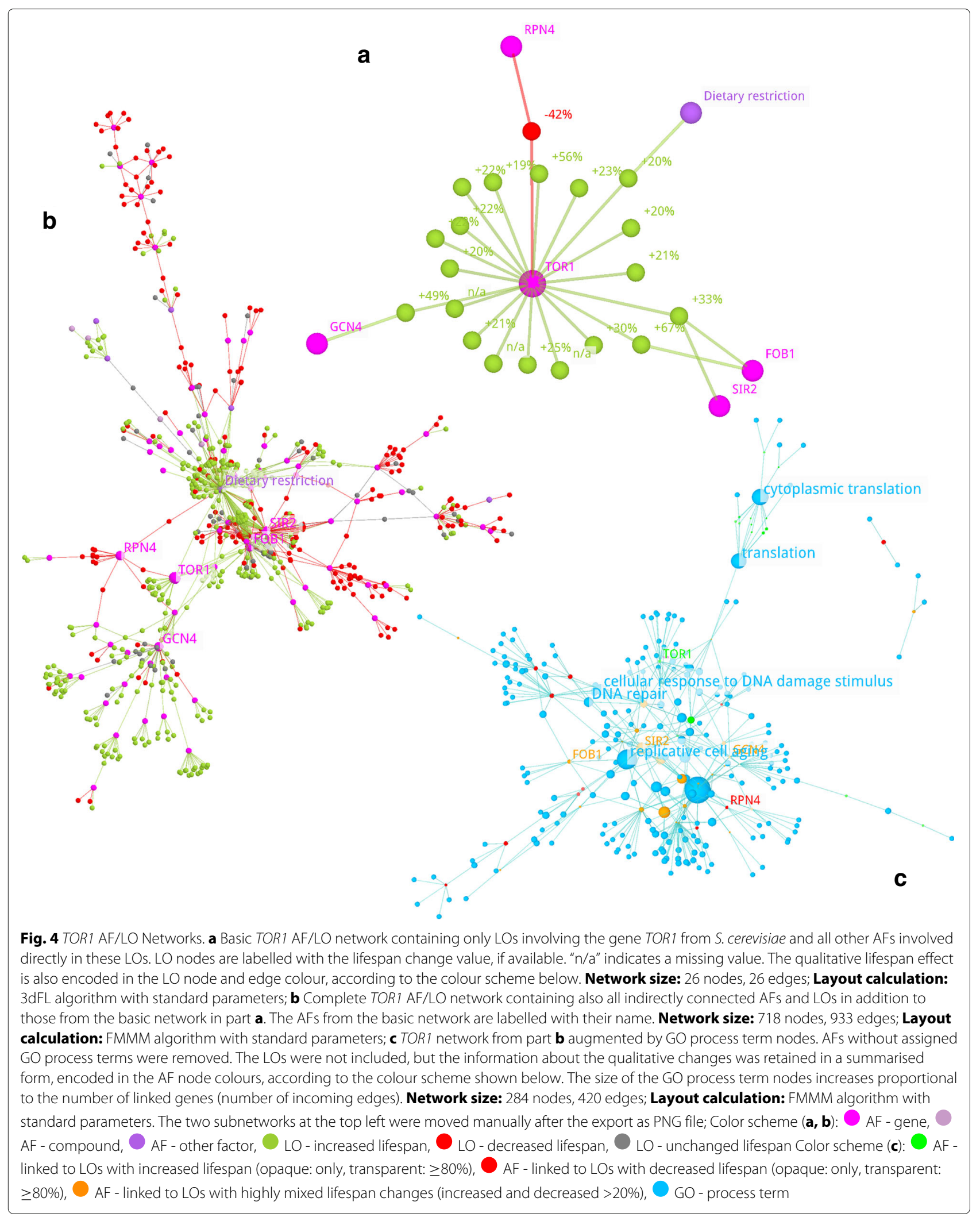


complete neighbourhood case. Figure $4 \mathrm{~b}$ shows the complete neighbourhood graph of TOR1. It can be seen as the largest connected subgraph including TOR1 and all directly or indirectly connected LOs and AFs for S. cerevisiae. The complete network consists of 718 nodes (78 AFs, 640 LOs) and 933 edges. The layout was built using the FMMM algorithm.

The extended graph reveals new information on RPN4. All experiments that included this gene led to a decreased lifespan. For GCN4, FOB1, and SIR2, the other direct neighbours of TOR1, there were observed decreased as well as increased lifespans.

The inclusion of all directly or indirectly connected LOs and AFs into the network enables to get an overview of all ageing factors examined directly or indirectly with TOR1. It would be much more laborious to compile the same dataset from the tabular AgeFactDB data and it would result in a very large table. In contrast to example 1 , the much larger network profits much more from the 3D view compared to a $2 \mathrm{D}$ view. To illustrate this, a $2 \mathrm{D}$ view of this network, generated with the popular 2D network viewer Cytoscape [25], is shown in Additional file 1: Figure S2 and a JANet stereo representation in Additional file 1: Figure S3. The advantage of the $3 \mathrm{D}$ view will become even more obvious comparing interactive views in JANet and Cytoscape.

\section{Example 3: augmentation via annotation nodes}

In the augmentation example shown here the direct neighbourhood network is augmented by allele type (AT) and citation (CI) nodes. The AT provides information about the experimental manipulation of a gene, for example deletion or overexpression. To increase the benefit of adding AT nodes, we unified the ATs according to Table 3. There are for example 12 different ATs which are unified to loss of function. The original AT information was kept as annotation of the $\mathrm{LO}$ nodes.

CI nodes provide information about the publication from which an LO was extracted, represented by the corresponding PubMed ID.

Figure 5a displays the basic network of the gene RAS2 from S. cerevisiae. It is homologous to members of the the mammalian RAS oncogene family, involved in the development of cancers [36]. There are 11 LOs where only the RAS2 gene is involved. The corresponding lifespan changes seem to be contradictory: 6 times an increased lifespan versus 5 times a decreased lifespan. In Fig. 5b the basic network is augmented by AT and CI nodes. Here, most of the supposed contradictions are resolved immediately. In all cases with reduced lifespan the RAS2 gene was deactivated (AT: loss of function). In 5 of 6 cases with increased lifespan it was overexpressed instead (AT: overexpression). So the differences here fit to the expectation that overexpression of a gene has the opposite
Table 3 Allele Type (AT) Unification

\begin{tabular}{|c|c|c|}
\hline AT & AT count & Unified AT \\
\hline $\begin{array}{l}\text { Conditional restoration } \\
\text { of Fgf23 activity in Fgf23 } \\
\text { knockout mice }\end{array}$ & 1 & Gain of function \\
\hline Gain of function & 27 & Gain of function \\
\hline Knockin & 2 & Gain of function \\
\hline Conditional knockout & 1 & Loss of function \\
\hline Deletion & 1013 & Loss of function \\
\hline Deletion / null & 1366 & Loss of function \\
\hline Deletion in connective tissue & 1 & Loss of function \\
\hline Deletion of a region & 1 & Loss of function \\
\hline Dominant negative & 27 & Loss of function \\
\hline Gene disruption & 1 & Loss of function \\
\hline Knock-down & 1 & Loss of function \\
\hline Knockdown & 2 & Loss of function \\
\hline Knockout & 67 & Loss of function \\
\hline Loss of function & 1631 & Loss of function \\
\hline Null mutant & 4 & Loss of function \\
\hline Non-null dominant & 3 & Mutation \\
\hline Non-null recessive & 93 & Mutation \\
\hline Non-null semi-dominant & 13 & Mutation \\
\hline Dominant negative mutation & 4 & Mutation \\
\hline Mutation & 135 & Mutation \\
\hline Mutation in adults & 1 & Mutation \\
\hline Mutations & 45 & Mutation \\
\hline Increased dosage & 1 & Overexpression \\
\hline Overexpression & 138 & Overexpression \\
\hline $\begin{array}{l}\text { Overexpression in cardiac } \\
\text { and skeletal muscles }\end{array}$ & 1 & Overexpression \\
\hline Overexpression in skin & 1 & Overexpression \\
\hline $\begin{array}{l}\text { Overexpression in stem and } \\
\text { progenitor cells }\end{array}$ & 1 & Overexpression \\
\hline $\begin{array}{l}\text { Overexpression of the short } \\
\text { isoform of p53 (p44) }\end{array}$ & 1 & Overexpression \\
\hline Over-expression & 381 & Overexpression \\
\hline $\begin{array}{l}\text { Pharmacological } \\
\text { overexpression (Superoxide } \\
\text { dismutase/catalase mimetics) }\end{array}$ & 1 & Overexpression \\
\hline RNA interference & 561 & RNA interference \\
\hline RNA interference and deletion & 1 & RNA interference \\
\hline RNA interference in adults & 2 & RNA interference \\
\hline $\begin{array}{l}\text { RNA interference post } \\
\text { development }\end{array}$ & 47 & RNA interference \\
\hline $\begin{array}{l}\text { RNA interference, } \\
\text { Knockdown }\end{array}$ & 1 & RNA interference \\
\hline RNAi knockdown & 1819 & RNA interference \\
\hline Anti-sense RNA & 1 & Anti-sense RNA \\
\hline $\begin{array}{l}\text { Deletion / null + RNAi } \\
\text { knockdown }\end{array}$ & 1 & $\begin{array}{l}\text { Deletionnull + } \\
\text { RNAi knockdown }\end{array}$ \\
\hline $\begin{array}{l}\text { Deletion / null + dominant } \\
\text { negative }\end{array}$ & 1 & $\begin{array}{l}\text { Deletion/null + } \\
\text { Dominant negative }\end{array}$ \\
\hline Deletion / null + normal & 2 & $\begin{array}{l}\text { Deletion / null + } \\
\text { normal }\end{array}$ \\
\hline $\begin{array}{l}\text { Deletion/null } \\
\text { over-expression }\end{array}$ & +1 & $\begin{array}{l}\text { Deletion / null + } \\
\text { over-expression }\end{array}$ \\
\hline Epigenetic modification & 2 & $\begin{array}{l}\text { Epigenetic } \\
\text { modification }\end{array}$ \\
\hline $\begin{array}{l}\text { Gain of function + loss of } \\
\text { function }\end{array}$ & 1 & $\begin{array}{l}\text { Gain of function + } \\
\text { loss of function }\end{array}$ \\
\hline $\begin{array}{l}\text { Germline ablation in } \\
\text { daf-2 mutants }\end{array}$ & 1 & $\begin{array}{l}\text { Germline ablation } \\
\text { in daf- } 2 \text { mutants }\end{array}$ \\
\hline
\end{tabular}


Table 3 Allele Type (AT) Unification (Continued)

\begin{tabular}{|c|c|c|}
\hline AT & AT count & Unified AT \\
\hline $\begin{array}{l}\text { Loss of function }+ \\
\text { RNAi } \\
\text { knockdown }\end{array}$ & 3 & Loss of function + RNAi knockdown \\
\hline $\begin{array}{l}\text { Loss of function }+ \\
\text { normal }\end{array}$ & 1 & Loss of function $+\quad$ normal \\
\hline $\begin{array}{l}\text { Loss of function + } \\
\text { over-expression }\end{array}$ & 6 & Loss of function + over-expression \\
\hline Normal & 31 & Normal \\
\hline $\begin{array}{l}\text { Overexpression } \\
\text { or mutations }\end{array}$ & 1 & Overexpression or mutations \\
\hline $\begin{array}{l}\text { Overexpression, } \\
\text { mutations, } \\
\text { deletion }\end{array}$ & 1 & Overexpression, mutations, deletion \\
\hline $\begin{array}{l}\text { Over-expression+ } \\
\text { over-expression }\end{array}$ & 2 & Over-expression + over-expression \\
\hline $\begin{array}{l}\text { Over-expression+ } \\
\text { unknown }\end{array}$ & 2 & Over-expression + unknown \\
\hline $\begin{array}{l}\text { RNA interference } \\
\text { and mutations }\end{array}$ & 1 & RNA interference and mutations \\
\hline $\begin{array}{l}\text { RNA interference, } \\
\text { Mutations }\end{array}$ & 1 & RNA interference, Mutations \\
\hline $\begin{array}{l}\text { Transient } \\
\text { expression }\end{array}$ & 1 & Transient expression \\
\hline Unknown & 447 & Unknown \\
\hline
\end{tabular}

The AT provides information about the experimental change applied to a gene, for example deletion or overexpression. To increase the benefit of adding AT nodes to networks the ATs were unified. There are for example 12 different ATs which are unified to loss of function. The upper part of the table, until the horizontal line, contains all unified ATs with at least 2 non-unified ATs. The AT count indicates the number of occurrences within AgeFactDB lifespan observations. The original AT information was kept as annotation of the LO nodes. The lower part of the table contains all otherATs, which were not unified

effect compared to loss of function. It can be seen that the remaining contradictory LO was extracted from a different publication than the others. In general, this could be a hint that the experiments might have been performed under different conditions, which were not recorded during the extraction of the LO. But in this case, we could not resolve the contradiction by studying the two publications.

This example demonstrates how helpful it can be to include annotation information like ATs and CIs as additional nodes. Potential inconsistencies can be resolved quickly, without having to look up and remember the annotations individually for each node. Because the annotation nodes influence the network layout they can also help to identify quickly characteristics of the examined ageing factors. For RAS2 such a characteristic is, that it seems to be a longevity promoting gene, meaning that its overexpression prolongs life while its inactivation shortens life.

\section{Example 4: augmentation combined with data transfer}

In this example the techniques Augmentation via Annotation Nodes and Data Transfer are combined within the following 2 steps.
Step1: The complete neighbourhood network of TOR1 from Fig. 4b was augmented with Gene Ontology (GO) terms [28]. The resulting intermediate visualisation is shown in Additional file 1: Figure S4.

The augmentation increased the network size by about thirty percent, from 718 to 947 nodes (78 AFs, 640 LOs, 229 GO terms) and from 933 to 1353 edges. A stereo representation of the intermediate visualisation can be found in Additional file 1: Figure S5.

Step2: The Data Transfer from the LOs to the AFs in the second step compensates the increased network complexity by allowing to remove all 640 LO nodes (Fig. 4c). Here, the average observed lifespan changes are indicated by the colours of AF nodes. Genes, for which all connected LOs increased or decreased the lifespan, are coloured in green or red. Genes, for which the effects of at least eighty percent of the LOs are going into the same direction, are coloured translucent green or red. Genes with even more heterogeneous LOs are coloured in orange.

The blue annotation nodes symbolize GO terms of molecular processes. The size of the nodes increases proportional to the number of linked genes (number of incoming edges).

The annotation nodes group connected components close to each other. While GO terms connected with several genes tend to build clusters within the network, GO terms related to a single gene build satellites at the outside of the network.

Some of the GO terms are assigned to a larger number of AFs, indicated by the node size. The labelled GO terms replicative cell aging (connected to 14 AFs), cellular response to DNA damage stimulus (connected to $6 \mathrm{AFs}$ ), and DNA repair (connected to AFs) reveal therefore a connection of many AFs in the network to cell ageing and DNA repair processes playing an important and widely accepted role in ageing.

This example demonstrates on one hand the combination of two visualisation techniques. On the other hand it shows how the increase in network size and complexity by the inclusion of GO annotation nodes can be compensated. And the stereo representation in Additional file 1: Figure S3 provides a good impression of the benefit of a 3D network layout.

\section{JANet use cases}

We provide use cases for the application of JANet. In the first use case, JANet is utilised for analysing a set of differentially expressed genes. In the second use case we demonstrate how JANet can be used for the identification of novel candidate genes related to ageing.

\section{Use case 1: analysis of differentially expressed genes}

JANet can be used to inspect user-specified gene lists within the LO networks extracted from AgeFactDB. 


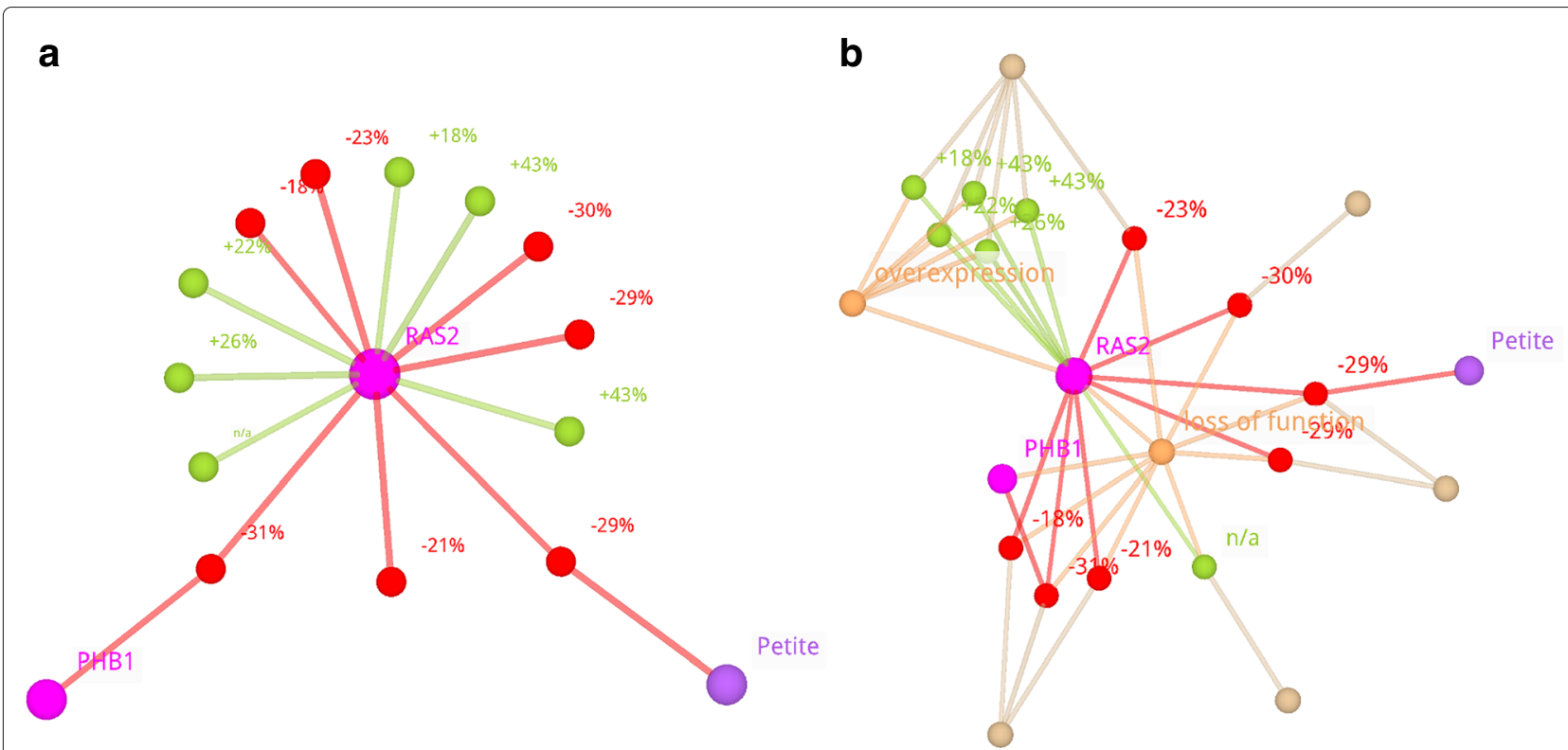

Fig. 5 Basic RAS2 AF/LO Network Augmented with AT and CI Nodes. Shown is the AF/LO network containing only LOs involving the gene RAS2 from S. cerevisiae and all AFs involved in these LOs (basic RAS2 network). LO nodes are labelled with the lifespan change value, if available. "n/a" indicates a missing value. The qualitative lifespan effect is also encoded in the LO node and edge colour, according to the colour scheme below. a There are 11 LOs were only the RAS2 gene is involved. But the lifespan change values seem to be contradictory: 6 times an increased lifespan versus 5 times a decreased lifespan. Network size: 16 nodes, 15 edges; Layout calculation: $3 \mathrm{dFL}$ algorithm with standard parameters; $\mathbf{b}$ In addition to the nodes in part $\mathbf{a}$, ATs and Cls from these LOs are included. ATs describe the experimental changes applied to genes, for example deletion or overexpression. They were unified according to Table 3. Citations resemble the publications from which the LOs were extracted, represented by the corresponding PubMed IDs. In contrast to the pure basic network in part A, most of the possible contradictions between the lifespan changes are resolved immediately. In all cases with reduced lifespan the RAS2 gene was deactivated (AT: loss of function). In 5 of 6 cases with increased lifespan it was overexpressed instead (AT: overexpression). So the differences here fit to the expectation that overexpression of a gene has the opposite effect compared to loss of function. This leaves a single contradiction. One can see that the remaining contradictory LO was extracted from a different publication than the others. In general, this could be a hint that the experiments might have been performed under different conditions, which were not recorded during the extraction of the LO. Network size: 23 nodes, 44 edges; Layout calculation: $3 \mathrm{dFL}$ algorithm with standard

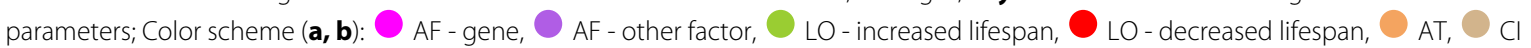

As an example we analyse differentially expressed genes taken from a study on the effect of D-Glucosamine $(\mathrm{GlcN})$ on the lifespan of nematodes and ageing mice by Weimer et.al. [37]. The study comprises RNA-seq data of $12 \mathrm{C}$. elegans samples and $12 \mathrm{M}$. musculus samples. For each species, 6 samples were treated with GlcN supplementation; the other samples remained untreated. Data are available in the NCBI Gene Expression Omnibus (GEO) database [38] under accession GSE54853.

In their experiments, Weimer et al. identified 293 differentially expressed genes in mouse liver and 1272 genes in C. elegans. We analyse the combined list with 1565 genes.

Figure 2c illustrates how the analysis is started within JANet:

1 Copy / paste the list of differentially expressed genes into the tab "Import Gene List" (The viewer needs either the ID from the NCBI Gene database [39] or the gene symbol plus species name to be able to match a gene to an $\mathrm{AF}$ or putative $\mathrm{AF}$.)
2 Start the analysis by clicking the "Start analysis" button.

The results are presented in a table containing the genes matching an AgeFactDB gene. Five entries of 157 matching gene entries are shown in Fig. 2d. For each gene the information provided by the user (NCBI Gene ID or gene symbol plus species name) and the corresponding information within AgeFactDB is shown. The table also provides the ageing relevance evidence type, characterising a matching gene as an ageing factor ("experimental evidence") or a putative ageing factor ("homology analysis"). For putative ageing factors the homologous non-putative ageing factors are specified.

A visualisation of the results is presented in Fig. 6. It provides an overall impression on the differentially expressed genes and their fit into the LOs in AgeFactDB. For each matched differentially expressed gene the LO network is shown (FMMM). Only matching genes or their homologues with experimental evidence are included. Other 


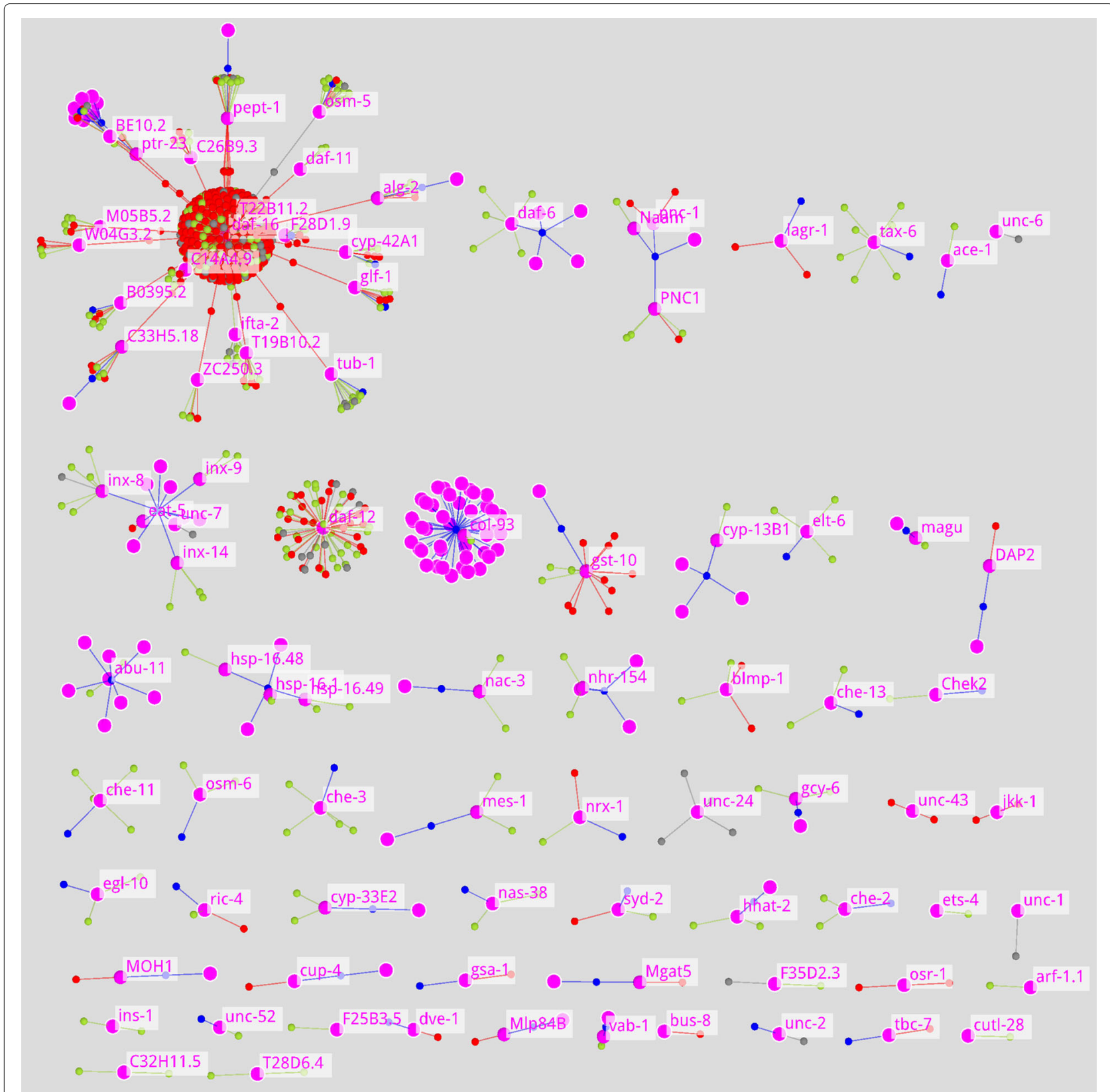

Fig. 6 Differentially Expressed Genes Matched to AF/LO Subnetworks in a Tiled View. Shown are all 60 AF/LO subnetworks containing at least one gene from the list of differentially expressed genes [37]. The homology observations are included in the subnetworks without genes that are not from the list of differentially expressed genes. The 151 differentially expressed genes are marked by a halo. Genes with experimental ageing relevance evidence are labelled with their gene symbol. The qualitative lifespan effect is encoded in the LO node and edge colour, according to the colour scheme below. Network size: 952 nodes, 898 edges; Layout calculation: FMMM algorithm with standard parameters; Color scheme: AF-gene, AF-compound, AF-other factor, $\bigcirc$ LO - increased lifespan, $\bigcirc$ LO-decreased lifespan, $\bigcirc$ LO-unchanged lifespan, observation - homology analysis, differentially expressed gene (gene of interest)

genes involved in the LOs were excluded to provide a clearer overview.

The network also includes the observations from the homology analysis. Again, non-matching genes belonging to the homology groups were excluded. The genes from the user specified list are marked by a halo. 151 genes with LOs are given in the graph.

The first subnetwork at the top left is very large and looks rather different. It is centred around the ageing factor daf-16 from $C$. elegans. For this gene 
many LOs are available (384). Most of them are concentrated in the sphere-like structure at the centre of the subnetwork. It was tested in combination with many other genes. A large number of AFs (17) and putative AFs (11) match to differentially expressed genes.

The second subnetwork is centred on the AF col-93 from C. elegans. It only contains a single LO and a homology observation with a large homology group of 42 putative AFs, arranged in a sphere-like structure. The AF col-93 is not in the user specified list. The third subnetwork centred on daf-12 contains no other genes but only $60 \mathrm{LOs}$ arranged in a sphere-like structure.

The other subnetworks have a rather similar structure: They consist of 1 to $5 \mathrm{AFs}$ with a at most $13 \mathrm{LOs}, 7$ putative $\mathrm{AFs}$, and 1 homology analysis observation. Several of the homology analysis observations are not connected to any putative AFs. This means that none of the other members of the homology group are on the user specified list.

Individual networks can be generated for each of the matching genes in the user specified list. An interactive table (Fig. 2d) can be used to narrow the number of AFs. Putative AFs are marked by a background colour in the interactive table.

The gene Gstp2 (Mus musculus) from the user specified list is an example for a putative AF in AgeFactDB. For this type of AF, no LOs are available in the database. The list from Fig. $2 \mathrm{~d}$ provides the homologous gene gst-10 from C. elegans for which LOs exist. Networks for gst-10 can be constructed via a dropdown list (Fig. 2a). The result is shown in Additional file 1: Figure S6.

In contrast to the overview network (Fig. 6), all AFs involved in the LOs are included. The network is augmented with AT and CI nodes. The numbers at the LO nodes indicate the lifespan change. It can be seen that overexpression of the gst-10 resulted in an increased lifespan by about 20 percent, while reducing the expression by RNA interference resulted in a decreased lifespan by about 12 percent. It can also be seen that this data was gathered from the experiments reported in 3 publications.

This use case demonstrates how easy you can identify AFs within a large list of genes with JANet. The overview network provides a compact view of all LOs available for these ageing factors. The tiling of disconnected subnetworks separates AFs studied more extensively in combination with other AFs from those studied separately. Individual genes can also easily be looked at in more detail by building individual subsets.

\section{Use case 2: candidate gene identification}

JANet can be used for a de novo candidate gene identification on the basis of AgeFactDB. The following section provides a show case for a possible selection process for C. elegans. The task will be the identification of new promising candidates for ageing-related genes that are not yet included in AgeFactDB.

An overview of the candidate gene selection process is given in Fig. 7. It consists of nine major steps. For each step there is also an enlarged image available as additional file (Additional file 2: Figures S7-S15).

Step1: We start our screening with the inspection of the complete LO network of C. elegans (step 1, Fig. 7a and Additional file 2: Figure S7). It consists of 965 ageingrelated genes and $4265 \mathrm{LOs}$ and is divided into 676 disjunct subnetworks.

Step 2: The LO network is augmented by the introduction of pathway nodes that represent KEGG pathways [29] specific for C. elegans. These pathways were extracted from the BioSystems database [40], based on cross-linking information to AFs. The other gene components of a KEGG pathway are introduced as additional gene nodes, linked also to the pathway node. AFs unconnected to a pathway node are removed. The resulting network is shown in Fig. $7 \mathrm{~b}$ and Additional file 2: Figure S8. This step reduced the numbers of ageing-related genes (324), LOs (2159) and disconnected subnetworks (1). A number of 2619 genes and 161 KEGG pathways were added. Linked to known AFs via common KEGG pathways, the additional genes can be seen as an initial set of candidates for ageing-related genes.

Step 3: A summary of the LO information was transferred to the AF nodes in preparation for reducing the complexity of the network (Fig. 7c and Additional file 2: Figure S9). This means that the LO node colours, indicating lifespan increase or decrease, from all LOs connected to an AF were transformed into a new colour for the AF node. The transformation was done by the following scheme:

- only red LOs $\rightarrow$ opaque red AF

- $\geq 80 \%$ red LOs $\rightarrow$ translucent red AF

- only green LOs $\rightarrow$ opaque green AF

- $\geq 80 \%$ green LOs $\rightarrow$ translucent green AF

- $>20 \%$ red and $>20 \%$ green LOs $\rightarrow$ orange AF

Step 4: The complexity of the network was reduced by hiding all 2159 LO nodes (Fig. 7d and Additional file 2: Figure S10).

Step 5: In order to focus on the most relevant AFs, the visible network was restricted to those AFs with a maximal lifespan change of at least $100 \%$. Only the corresponding connected pathways and additional genes are shown (Fig. 7e and Additional file 2: Figure S11). It consists now of 768 additional genes connected to 46 pathways.

Step 6: Based on the assumption that genes which are connected to AFs via multiple pathways are more likely to be ageing-related, promising candidates were 


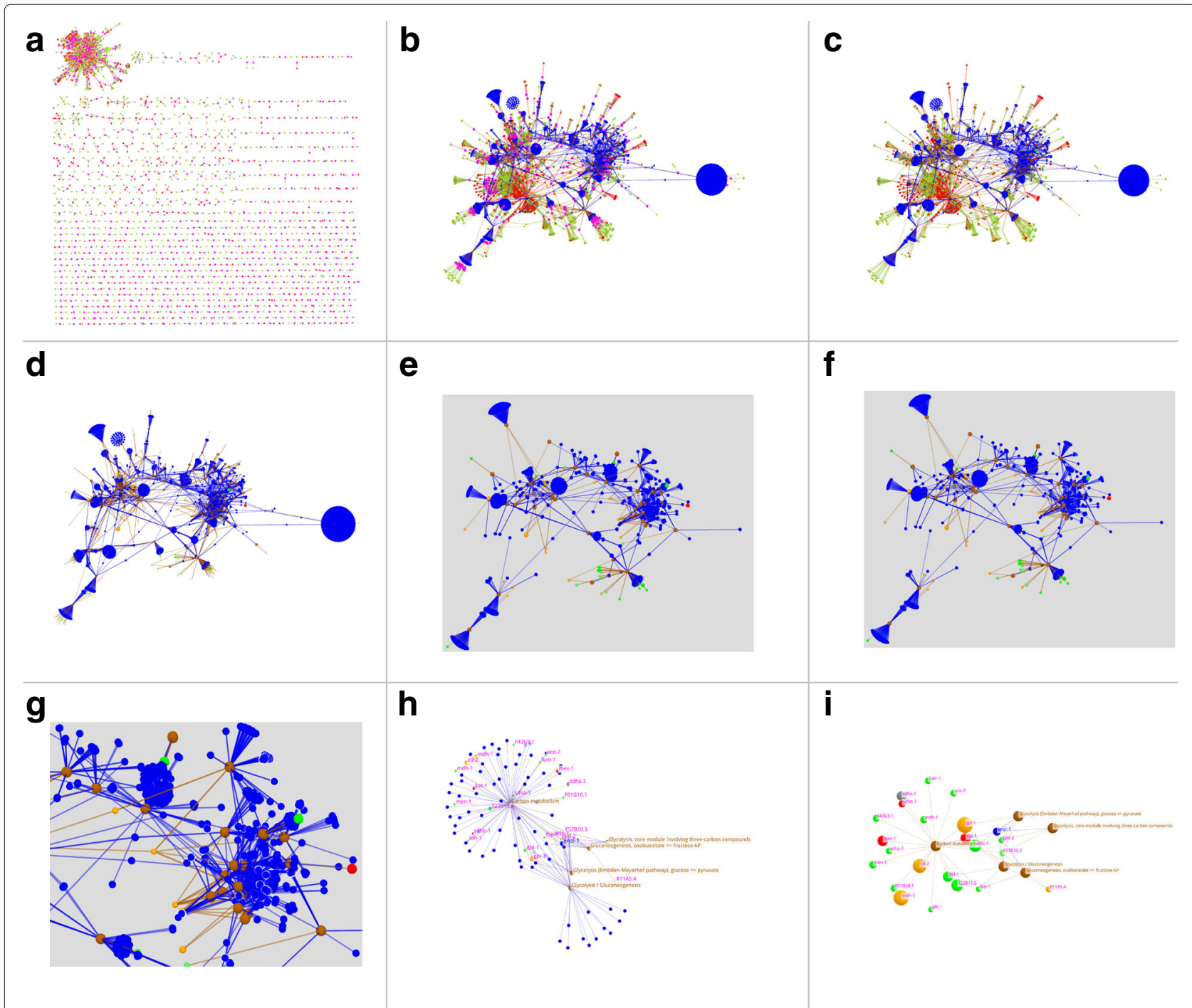

Fig. 7 Candidate Gene Identification - Overview. Shown is an overview of the visualisation strategy to identify new candidate genes of C. elegans for inclusion in the database AgeFactDB. For each part of this figure there is also a larger version available, as an individual figure with a more detailed description (Additional file 2: Figures S7-S15). In the combined colour scheme below it is indicated which colours are applicable to which part. The layout was calculated in step 1 and 2 using the FMMM algorithm. Throughout step 3 to 8 the layout from step 2 was used, to facilitate tracking the changes. Instead of changing the layout, nodes were hidden, or node attributes like colour or size were changed during these steps. In step 9 a new layout was calculated using the FR algorithm. a Step 1: the complete AF/LO network of C. elegans with 675 subnetworks as starting point (Additional file 2: Figure S7); b Step 2: augmentation with KEGG pathway and gene nodes, based on cross-linking information from the BioSystems database (Additional file 2: Figure S8); c Step3: visual transfer of lifespan change information from LO nodes to AF nodes (Additional file 2: Figure S9); d Step 4: reduction of network complexity by hiding all LO nodes (Additional file 2: Figure S10); e Step 5: first reduction of candidate gene number by applying a minimum lifespan change limit (Additional file 2: Figure S11); f Step 6: second reduction of candidate gene number by selecting only genes connected to at least 6 KEGG pathway nodes; final selected candidate genes are marked by a halo (Additional file 2: Figure S12); $\mathbf{g}$ Step 7: zoom into the area with most of the candidate genes; Table 4 lists the final selected candidate genes and their literature analysis results (Additional file 2: Figure S13); $\mathbf{h}$ Step 8: focus on candidate gene enol-1 by zooming and selective node display (Additional file 2: Figure S14); i Step 9: visualisation of the basic pathway network of candidate gene enol-1, augmented by AFs with data transferred from their LOs (Additional file 2: Figure S15); Color

scheme: AF-gene (a,b), AF-compound (a), AF-other factor (a), AF - LOs with increased lifespan (opaque: only, transparent: $\geq 80 \%$; $\mathbf{c}, \mathbf{d}, \mathbf{e}, \mathbf{f}, \mathbf{g}, \mathbf{h}, \mathbf{i}), \mathrm{AF}$ - LOs with decreased lifespan (opaque: only, transparent: $\geq 80 \% ; \mathbf{c}, \mathbf{d}, \mathbf{i}), \mathrm{AF}$ - LOs with highly mixed lifespan changes (increased and decreased $>20 \% ; \mathbf{c}, \mathbf{d}, \mathbf{e}, \mathbf{f}, \mathbf{g}, \mathbf{h}, \mathbf{i}), \bigcirc \mathrm{LO}$ - increased lifespan (a,b,c small), $\mathrm{LO}$ - decreased lifespan (a,b,c small), LO - unchanged lifespan $(\mathbf{a}, \mathbf{b}, \mathbf{c}), \quad$ KEGG pathway $(\mathbf{b}, \mathbf{c}, \mathbf{d}, \mathbf{e}, \mathbf{f}, \mathbf{g}, \mathbf{h}, \mathbf{i}), \bigcirc$ gene (from pathway and not in AgeFactDB; $\mathbf{b}, \mathbf{c}, \mathbf{d}, \mathbf{e}, \mathbf{f}, \mathbf{g}, \mathbf{h})$, 
Table 4 C. elegans candidate genes

\begin{tabular}{ll}
\hline Gene symbol & NCBI \\
\hline acdh-7, acdh-8 & 18175 \\
aco-1 & 18132 \\
& \\
alh-4 & 17902 \\
acs-3 & 17867 \\
acs-4 & 17600 \\
acs-17 & 18085
\end{tabular}

$\begin{array}{lr}\text { dbl-1 } & 179068 \\ \text { ech-6 } & 176376 \\ \text { ech-9 } & 184065 \\ \text { enol-1 } & 174423 \\ & \\ \text { F08F8.7 } & 176043 \\ \text { F54C8.1 } & 186222 \\ \text { F59F4.1 } & 181668 \\ & \\ \text { hacd-1 } & 178638 \\ \text { idhg-2 } & 172430 \\ \text { mek-1 } & 181004 \\ \text { pfk-1.2 } & 179335 \\ \text { pkc-2 } & 18166\end{array}$

Ageing relevance evidence

Expression of human homologous gene ACADM decreases over age in skin [45].

Mutant animals lacking genes aco-1 and ftn-1 show significant reduced lifespan upon iron stress, while N2 and ftn-2 animals show no difference. The results suggest that ftn-1 and aco-1 are transcriptionally regulated by iron and are important for iron homeostasis affecting lifespan upon iron stress conditions in C. elegans [42].

Gene alh-4 is involved in determination of adult lifespan [46].

acs-3 mutants exhibited a significantly shorter lifespan on E. coli OP50 (12.8 days), as compared to WT animals (17.9 days) [52].

RNAi of acs-4 resulted in a 6.1\% increase in lifespan compared to wildtype [53].

Many "dod" genes are responsible for the longevity of daf-2 mutants. The "dod" genes that could previously be identified as life span regulating genes include among others dod-9/acs-17 [54,55].

Metabolic fingerprint studies with long-lived mutants daf-2 and eat-2 showed strong upregulation of enzymes involved in alcohol fermentation including alh-5, alh-12, sodh-1 and sodh-2 [56].

C. elegans mutants with a loss-of-function in dbl-1 showed reduced lifespan [57].

Metabolism of short-chain organic acids (e.g. gene ech-6) is upregulated in states of impaired IGF-1 signalling in a daf-2 mutant strain with extended lifespan [47].

The top five overrepresented categories yielded by the comparative expression analysis in three ets-4 deletion strains with increased lifespan include fatty acid metabolic process genes (acdh-2, ech-9, and C48B4.1) [48].

Compared to wild type strain N2 the RNAi knockdown of gene enol-1 reduced the mean lifespan by $11-14 \%$. Compared to eat-2 knockout strains it reduced the mean lifespan by $15-19 \%$ in strain eat-2(ad1116) and by $28 \%$ in strain eat2(ad465) [41].

F08F8.7 was found to be upregulated in long-living mutant daf-2 in comparison to N2 [56].

Concerning branched-chain amino acid metabolism, F54C8.1 was upregulated in the long-lived mutant daf-2 [56]

Gene F59F4.1 was linked to Parkinson's disease by screens involving alphasynuclein. In alpha-synuclein expressing nematode lines age dependent degeneration of dopaminergic neurons was observed [49].

Gene hacd-1 was linked to lifespan effects of germline mutants by transcriptome analysis [50].

Energy metabolism seems to be differentially regulated in long-lived mutants compared to N2. Particularly idhg-2 was upregulated in daf-2 mutants [56].

The lifespan extending effect of intermittent feeding (IF) of $74.9 \%$ in wiltype strain $\mathrm{N} 2$ is reduced to $21.7 \%$ in the mek-1 loss-of-function strain ks54 [58].

Long-living mutants show upregulation of $p f k-1.2$ a gene encoding for one of the key enzymes of glycolysis [56].

Knockout of gene $p k c-2$ decreased the mean lifespan significantly by $3-5 \%$ at $15^{\circ} \mathrm{C}$ and compensated for the short-lived phenotype of trpa-1 mutant worms at $20^{\circ} \mathrm{C}$. Overexpression of gene $p k c-2$ increased the mean lifespan significantly by $3-6 \%$ at $20^{\circ} \mathrm{C}[43]$.

The youthful swimming phenotype of let-23 ( $g f$ ) mutant was suppressed by RNAi knockdown of plc-3 and itr-1 [59].

Compared to wildtype strain N2 the RNAi knockdown of gene pyk-1 reduced the mean lifespan by $19-21 \%$. And compared to eat-2 knockout strain eat-2(ad1116) it reduced the mean lifespan by 19-29\% [41].

The traditional Chinese medicine Gengnianchun (GNC) prolongs the lifespan of C. elegans. Comparison of genome-wide transcriptional profiling of untreated and GNC treated worms at day 22 showed that GNC downregulated the expression of R04A9.7 [60]. 
Table 4 C. elegans candidate genes (Continued)

\begin{tabular}{|c|c|c|}
\hline Gene symbol & NCBI gene ID & Ageing relevance evidence \\
\hline$r b x-1$ & 179358 & RNAi of $r b x-1$ results terminal stage arrest during embryogenesis[61]. \\
\hline sdhd-1 & 174692 & Extension of lifespan in gas-1 (fc21) was observed after sdhc-1 and sdhd-1 knockdown [62]. \\
\hline skr-9 skr-14 sma-4 & 178494178839175815 & $\begin{array}{l}\text { Wnt signaling is highly involved in the aging process of C. elegans with a shifting dynamic. From L4 } \\
\text { to D6 Wnt signalling is upregulated and from D6 to D15 it is down-regulated. Eight genes including } \\
\text { skr-9, skr-14, and sma-4, which are involved in the Wnt signalling, were significantly up-regulated } \\
\text { from L4 to D6 and down-regulated from D6 to D15 [63]. }\end{array}$ \\
\hline sucg-1, sucl-2 & 177555175252 & $\begin{array}{l}\text { The decrease of female reproductive senescence is a hallmark of ageing. The inactivation of sucg-1 } \\
\text { or sucl-2 by RNAi extends the reproductive lifespan [64]. }\end{array}$ \\
\hline T02G5.7 & 3565206 & The gene kat-1, a parolog of gene T02G5.7, is included as AF AF_006931 in AgeFactDB [51]. \\
\hline tpi-1 & 174844 & $\begin{array}{l}\text { RNAi knockdown of gene tpi- } 1 \text { reduced the the mean lifespan by } 8 \% \text { and the maximum lifespan by } \\
10 \%[44] \text {. }\end{array}$ \\
\hline
\end{tabular}

Candidate genes obtained by the candidate gene selection strategy summarised in Fig. 7. Results of the literature search for ageing relevance evidence. No specific ageing relevance evidence was found for the following genes: acox-1, acox-3, acox-5, acs-2, acs-13, acs-15, acs-16, acs-18, acs-19, acs-23, agxt-1, aldo-2, alh-8, alh-9, B0272.3, C30H6.7, C44H4.6, C50D2.7, D2063.1, dlst-1, ech-8, F08A8.4, F11F1.1, F44E5.4, F44E5.5, got-1.3, got-2.1, got-2.2, gstk-1, hsp-70, hxk-1, idhg-1, ist-1, pdhb-1, pfk-1.1, pgk-1, R03D7.5, R05F9.6, rpia-1, skr-4, skr-6, skr-10, skr-16, skr-17, skr-18, skr-21, suca-1, sucl-1, tpi-1, ugt-23, ugt-46, ugt-48, ugt-50, ugt-55, ugt-56, ugt-58, ugt-61, ugt-62, Y105E8B.9, Y43F4B.5, Y71G12B.10

highlighted. Only those genes connected to at least 6 pathway nodes were selected and marked by a halo (Fig. $7 \mathrm{f}$ and Additional file 2: Figure S12). This led to a final list of 95 candidate genes connected to 20 visible pathway nodes.

Step 7: A more detailed view was created by zooming in at the position with the largest number of highlighted candidate genes (Fig. 7g and Additional file 2: Figure S13).

Step 8: It is also possible to reinspect the network while focusing on a single candidate gene. As an example gene enol-1 was used (Fig. 7h and Additional file 2: Figure S14). We revisited the network before the reduction of the pathway and gene nodes (step 4), while focusing on enol-1. All nodes were hidden that are not connected to the 7 pathway nodes which are connected to enol-1. The candidate gene node, the AF nodes, and the pathway nodes are labelled with their names.

Step 9: An optimized view was created for candidate gene enol-1 by building a new subset and calculating a new layout (Fig. 7i and Additional file 2: Figure S15). The new subset contains enol-1, all KEGG pathway nodes connected to it, and all AFs connected to these pathway nodes. Like before, information from the LOs was transferred to the AFs and visualized by node colour and size. As you can see, enol-1 is linked to 3 different types of pathways: biosynthesis, degradation and energy metabolism. The LOs were obtained in the context of energy metabolism [41].

For all 95 selected candidate genes obtained in step 6 we did a literature search. The results are summarized in Table 4. LOs not yet contained in AgeFactDB with a significant lifespan change are available for the 5 candidate genes aco-1, enol-1, pkc-2, pyk-1, and tpi-1 [41-44]. Other ageing relevance evidence was found for the 8 other candidate genes acdh-7, acdh-9, alh-4, ech-6, ech-9, F59F4.1, hacd-1, and T02G5.7 [45-51]. No specific ageing relevance evidence was found for the remaining 35 candidate genes.

In this use case JANet helped to find new candidate genes as AFs for AgeFactDB. By applying several of the proposed visualisation techniques we could select 95 promising candidate genes from the initial 2619 genes. The literature search revealed for $27 \%$ of the candidate genes that there is ageing-relevance evidence. This true positive rate suggests that it would be justified to include also others of the remaining 35 candidate genes into experimental analysis. Moreover, such an insilico approach may also improve the curation of $\mathrm{AF} / \mathrm{LO}$ databases.

\section{Conclusion}

JANet provides a wide range of network visualisations for the analysis of lifespan observations. Integrating heterogeneous data from various sources, these networks allow a comprehensive overview of data from lifespan experiments and their dependencies. The investigations are linked via common components or external domain knowledge into network representations. This can generate interpretable patterns, which are recognisable by a human expert. These network representations can be seen therefore as a valuable addition to classic tabularized representations.

Interactive network manipulation allows replacing complex static queries with a visually guided search process. A life scientist can easily explore a network by merely changing the graph's perspective. For example, zooming into a region of interest can reveal detailed information, which might be hidden at a broader scale. In this context, interactive 3D layouts allow a more extensive range of manipulations than classical $2 \mathrm{D}$ graphs. 
Providing more compact graph representations and 3D rotation, 3D arrangements allow reaching distant points much faster. The network itself can be reconfigured during the exploration. For example, LOs can be hidden or highlighted.

In our first use case, we show that a researcher can utilise the network visualisations of AgeFactDB to explore his/her experimental data. This type of analysis brings a set of candidate genes into the context of thousands of LOs. In this way, the single experiment gets highly connected to the ageing research field, which would be more laborious by a traditional literature or database screen. This general ability of network representations of reflecting existing knowledge and facilitating the analysis of experimental data can be useful in many other research areas.

\section{Additional files}

Additional file 1: Figure S1: Large AF/LO Network Navigation in a 3D and 2D Network Viewer. Figure S2: The Complete TOR1 AF/LO Network as 2D View. Figure S3: The Complete TOR1 AF/LO Network as JANet Stereogram. Figure S4: The Complete TOR1 AF/LO Network Augmented with GO Process Term Nodes. Figure S5: A Stereogram of the Complete TOR1 AF/LO Network Augmented with GO Process Term Nodes. Figure $\mathbf{S 6}$ The Differentially Expressed Gene Gstp2 Matched to the AF/LO Subnetwork of Gene gst-10. (PDF 15,960 kb)

Additional file 2: Figure S7: Candidate Gene Selection - Step 1. Figure S8 Candidate Gene Selection - Step 2. Figure S9: Candidate Gene Selection Step 3. Figure S10: Candidate Gene Selection - Step 4. Figure S11: Candidate Gene Selection - Step 5. Figure S12: Candidate Gene Selection - Step 6. Figure S13: Candidate Gene Selection - Step 7. Figure S14 Candidate Gene Selection - Step 8. Figure S15: Candidate Gene Selection - Step 9. (PDF $8189 \mathrm{~kb})$

\section{Abbreviations}

3dFL: 3D forced layout; AF: Ageing factor; AgeFactDB: JenAge Ageing Factor Database; AT: Allele type; Cl: Citation; C. elegans: Caenorhabditis elegans; FR: Fruchterman-Reingold algorithm; FMMM: Fast multipole multilevel method; GEO: Gene Expression Omnibus; GO: Gene ontology; JANet: Jmol AgeFactDB network-viewer; LO: Lifespan observation; M. musculus: Mus musculus; S. cerevisiae: Saccharomyces cerevisiae

\section{Funding}

The work described here is part of a joint effort of the Research Cores (Forschungskern) JenAge and SyStaR within the Gerontosys II funding line. We acknowledge JenAge and SyStaR funding by the German Ministry for Education and Research (Bundesministerium für Bildung und Forschung, BMBF; JenAge support code: 0315581; SyStaR, support code: 0315894A). The research leading to these results has also received funding from the European Community's Seventh Framework Programme (FP7/2007-2013) under grant agreement n602783, the German Research Foundation (DFG, SFB 1074 project Z1), and the Federal Ministry of Education and Research (BMBF, e:Med, SYMBOL-HF, ID 01ZX1407A) to HAK.

\section{Availability of data and materials}

The data was taken from the public databases AgeFactDB (http://agefactdb. jenage.de), BioSystems (http://www.ncbi.nlm.nih.gov/biosystems), NCBI Gene (http://www.ncbi.nlm.nih.gov/gene), and Gene Ontology (http://www. geneontology.org). JANet is available at: http://sysbio.uni-ulm.de/software/ janet/. It can be started directly from this server. The complete source code is also available from this site. JANet requires a computer with at least 4 GB RAM and a modern web browser with Javascript activated.

\section{Authors' contributions}

$\mathrm{RH}$ and HAK conceived the study. VK, AF, RH implemented the methods and developed the web interface. RH, LL, MP, JS wrote the manuscript. SK and RH analysed the data. LL, HAK guided the work and revised the manuscript. All authors read and approved the final version of the manuscript.

\section{Ethics approval and consent to participate}

Not applicable.

\section{Consent for publication}

Not applicable.

\section{Competing interests}

The authors declare that they have no competing interests.

\section{Publisher's Note}

Springer Nature remains neutral with regard to jurisdictional claims in published maps and institutional affiliations.

\section{Author details}

${ }^{1}$ Institute of Medical Systems Biology - Ulm University, Albert-Einstein-Allee 11, 89081 Ulm, Germany. ${ }^{2}$ Leibniz Institute on Aging - Fritz Lipmann Institute, Beutenbergstr. 11, 07745 Jena, Germany. ${ }^{3}$ Institute of Neural Information Processing - Ulm University, Albert-Einstein-Allee 11, 89081 Ulm, Germany.

Received: 10 October 2017 Accepted: 25 September 2018

Published online: 23 October 2018

\section{References}

1. Hansen M, Hsu A-L, Dillin A, Kenyon C. New genes tied to endocrine, metabolic, and dietary regulation of lifespan from a caenorhabditis elegans genomic rnai screen. PLoS Genet. 2005;1 (1):17-01190128.

2. Berman RJ, Kenyon C. Germ-cell loss extends c. elegans life span through regulation of daf- 16 by kri-1 and lipophilic-hormone signaling. Cell. 2006;124(5):1055-68.

3. Kestler HA, Müller A, Gress TM, M B. Generalized venn diagrams: a new method of visualizing complex genetic set relations. Bioinformatics. 2005;21(8):1592-5.

4. Kestler HA, Müller A, Buchholz M, Gress TM, Palm G. A perceptually optimized scheme for visualizing gene expression ratios with confidence values. In: André E, Dybkjær L, Minker W, Neumann H, Weber M, editors. Perception and Interactive Technologies. Berlin: Springer; 2006. p. 73-84.

5. Müller A, Holzmann $K$, Kestler HA. Visualization of genomic aberrations using affymetrix snp arrays. Bioinformatics. 2007;23(4):496-7.

6. Kestler HA, Müller A, Kraus JM, Buchholz M, Gress TM, Liu H, Kane DW, Zeeberg BR, Weinstein JN. Vennmaster: area-proportional euler diagrams for functional go analysis of microarrays. BMC Bioinforma. 2008;9:67.

7. Gehlenborg N, Wong B. Into the third dimension. Nat Methods. 2012;9(9):851.

8. Cohen RF, Eades P, Lin T, Ruskey F. Three-dimensional graph drawing. Algorithmica. 1997;17(2):199-208.

9. Nakayama K, Siverman GH. Serial and parallel processing of visual feature conjunctions. Nature. 1986;320(6059):264-5.

10. Enns JT, Rensink RA. Influence of scene-based properties on visual search. Science. 1990;247(4943):721-3.

11. Phillips WA, Christie DFM. Components of visual memory. Q J Exp Psychol. 1977;29(1):117-33.

12. Xu Y, Nakayama K. Visual Short-Term Memory Benefit for Objects on Different 3-D Surfaces. J Exp Psychol Gen. 2007;136(4):653-62.

13. Munzner T. Rules of thumb. In: Munzner T, editor. Visualization Analysis \& Design. Boca Raton: A K Peters/CRC press; 2015. p. 116-144.

14. Hühne R, Thalheim T, Sühnel J. AgeFactDB - The JenAge Ageing Factor Database - Towards data integration in ageing research. Nucleic Acids Res. 2014;42(Database issue):892-6.

15. NCBI Resource Coordinators. Database resources of the National Center for Biotechnology Information. Nucleic Acids Res. 2015;43(Database issue): 6-17. http://www.ncbi.nlm.nih.gov/homologene. Accessed 03 Nov 2017.

16. AgeFactDB Lifespan Observation OB_000094. http://agefactdb.jenage. de/cgi-bin/jaDB.cgi? RKEY=r001\&SEARCH=OB_000094\&TYPE = d_ob\&VIEW=detail. Accessed 03 Nov 2017.

17. Fabrizio $\mathrm{P}$, Longo VD. The chronological life span of Saccharomyces cerevisiae,. Aging Cell. 2003;2:73-81. 
18. Mortimer RK, Johnston JR. Life Span of Individual Yeast Cells. Nature. 1959;183:1751-2

19. Hagberg AA, Schult DA, Swart PJ. Exploring network structure dynamics, and function using NetworkX. In: Varoquaux G, Vaught T, Millman J, editors. Proceedings of the 7th Python in Science Conference(SciPy2008). 2008. p. 11-5.

20. Csardi G, Nepusz T. The igraph software package for complex network research. Inter Journal. 2006;Complex Systems(1695):1-9.

21. vis.js - A dynamic, browser based visualization library. http://visjs.org Accessed 03 Nov 2017.

22. Rohn H, Junker A, Hartmann A, Grafahrend-Belau E, Treutler H, Klapperstück M, Czauderna T, Klukas C, Schreiber F. VANTED v2: a framwork for systems biology applications. BMC Syst Biol. 2012;6:139.

23. Cox CK, Eick GS, He T. 3D Geographic Network Displays. ACM SIGMOD Record. 1996;25(4):50-4

24. Cy3D. Simple 3D Network Renderer App. http://apps.cytoscape.org/ apps/cy3d. Accessed 03 Nov 2017

25. Shannon P, A M, Ozier O, Baliga NS, Wang JT, Ramage D, Amin N, Schwikowski B, Ideker T. Cytoscape: a software environment for integrated models of biomolecular interaction networks. Genome Research. 2003;13(11):2498-504.

26. Theocharidis A, van Dongen S, Enright AJ, Freeman TC. Network visualization and analysis of gene expression data using BioLayout Express $^{3 \mathrm{D}}$. Nature Protocols. 2009;4(10):1535-50.

27. Hachul S, Jünger M. Large-graph layout with the fast multipole multilevel method. Technical report. Cologne: University of Cologne, Computer Science Department; 2005. http://e-archive.informatik.uni-koeln.de/509/.

28. Gene Ontology Consortium. Creating the gene ontology resource: design and implementation. Genome Res. 2001;11(8):1425-33.

29. Kanehisa M, Goto S, Sato Y, Kawashima M, Furumichi M, Tanabe M. Data, information, knowledge and principle: back to metabolism in KEGG. Nucleic Acids Res. 2014:42(Database issue):199-205.

30. Wiki WP. Main Page — WebGL Public Wiki.

31. Danchilla B. Three.js Framework. Berkeley: Apress; 2012, pp. 173-203.

32. Van Bruggen R. Learning Neo4j. Birmingham: Packt Publishing; 2014.

33. Verlet L. Computer "experiments" on classical fluids. i. thermodynamical properties of lennard-jones molecules. Phys Rev. 1967;159:98-103.

34. Fruchterman TMJ, Reingold EM. Graph drawing by force-directed placement. Softw: Pract Experience. 1991;21(11):1129-64.

35. Evans DS, Kapahi P, Hsueh W-C. TOR signaling never gets old: Aging, longevity and TORC1 activity. Ageing Res Rev. 2011;10(2):225-37.

36. Powers S, Kataoka T, Fasano O, Goldfarb M, Strathern J, Wigler M. Genes in S. cerevisiae Encoding Proteins with Domains Homologous to the Mammalian ras Proteins. Cell. 1984;36(3):607-12.

37. Weimer S, Priebs J, Kuhlow D, Groth M, Priebe S, Mansfeld J, Merry TL, Dubuis S, Laube B, Pfeiffer AF, Schulz TJ, Guthke R, Platzer M, Zamboni N, Zarse K, Ristow M. D-glucosamine supplementation extends life span of nematodes and of ageing mice. Nat Commun. 2014;5:3563.

38. Barrett T, Wilhite SE, Ledoux P, Evangelista C, Kim IF, Tomashevsky M, Marshall KA, Phillippy KH, Sherman PM, Holko M, Yefanov A, Lee H, Zhang N, Robertson CL, Serova N, Davis S, Soboleva A. NCBI GEO: archive for functional genomics data sets-update. Nucleic Acids Res. 2013;41(Database issue):991-5.

39. Brown GR, Hem V, Katz KS, Ovetsky M, Wallin C, Ermolaeva O, Tolstoy I, Tatusova T, Pruitt KD, Maglott DR, Murphy TD. Gene: a gene-centered information resource at NCBI. Nucleic Acids Res. 2015;43(Database issue): 36-42.

40. Geer LY, Marchler-Bauer A, Geer RC, Han L, He J, He S, Liu C, Shi W, Bryant SH. The NCBI BioSystems database. Nucleic Acids Res. 2010;38(Database issue):492-6.

41. Yuan Y, Kadiyala CS, Ching TT, Hakimi P, Saha S, Xu H, Yuan C, Mullangi V, Wang L, Fivenson E, Hanson R, Ewing R, Hsu A, Miyagi M, Feng Z. Enhanced energy metabolism contributes to the extended life span of calorie-restricted Caenorhabditis elegans. J Biol Chem. 2012;287(37): 31414-26.

42. Kim Yl, Cho JH, Yoo OJ, Ahnn J. Transcriptional regulation and life-span modulation of cytosolic aconitase and ferritin genes in C. elegans. J Mol Biol. 2004;342(2):421-33.

43. Xiao R, Zhang B, Dong Y, Gong J, Xu T, Liu J, Xu XZ. A genetic program promotes $C$. elegans longevity at cold temperatures via a thermosensitive TRP channel. Cell. 2013;152(4):806-17.
44. Ralser M, Wamelink MM, Kowald A, Gerisch B, Heeren G, Struys EA, Klipp E, Jakobs C, Breitenbach M, Lehrach H, Krobitsch S. Dynamic rerouting of the carbohydrate flux is key to counteracting oxidative stress. J Biol. 2007;6(4):10.

45. Digital Ageing Atlas. Human Gene ACADM. http://ageing-map.org/atlas/ gene/34/ Accessed 03 Nov 2017.

46. WormBase. Caenorhabditis elegans Gene alh-4. http://www.wormbase. org/species/c_elegans/gene/WBGene00000110 Accessed 03 Nov 2017.

47. Zarse K, Schmeisser S, Groth M, Priebe S, Beuster G, Kuhlow D, Guthke R, Platzer M, Kahn CR, Ristow M. Impaired insulin/IGF1 signaling extends life span by promoting mitochondrial L-proline catabolism to induce a transient ROS signal. Cell Metab. 2012;15(4):451-65.

48. Thyagarayan B, Blaszczak AG, Chandler KJ, Watts JL, Johnson WE, Graves BJ. ETS-4 is a transcriptional regulator of life span in Caenorhabditis elegans. PLoS Genet. 2010;6(9):1001125.

49. Wolozin B, Gabel C, Ferree A, Guillily M, Ebata A. Watching worms whither: modeling neurodegeneration in C. elegans. Prog Mol Biol Transl Sci. 2011;100:499-514.

50. Ackerman D, Gems D. The mystery of C. elegans aging: An emerging role for fat. Distant parallels between C. elegans aging and metabolic syndrome?. Bioessays. 2012;34(6):466-71.

51. Soukas AA, Carr CE, Ruvkun G. Genetic regulation of Caenorhabditis elegans lysosome related organelle function. PLoS Genet. 2013;9(10): 1003908.

52. Ward JD, Mullaney B, Schiller BJ, He LD, Petnic SE, Couillault C, Pujol N, Bernal TU, Van Gilst MR, Ashrafi K, et al. Defects in the c. elegans acyl-coa synthase, acs-3, and nuclear hormone receptor, nhr-25, cause sensitivity to distinct, but overlapping stresses. PLoS One. 2014;9(3):92552.

53. Reis-Rodrigues P, Czerwieniec G, Peters TW, Evani US, Alavez S, Gaman EA Vantipalli M, Mooney SD, Gibson BW, Lithgow GJ, et al. Proteomic analysis of age-dependent changes in protein solubility identifies genes that modulate lifespan. Aging Cell. 2012;11(1):120-7.

54. Shaw WM, Luo S, Landis J, Ashraf J, Murphy CT. The C. elegans tgf- $\beta$ dauer pathway regulates longevity via insulin signaling. Curr Biol. 2007;17(19):1635-45

55. Murphy CT, McCarroll SA, Bargmann Cl, Fraser A, Kamath RS, Ahringer J, $\mathrm{Li} \mathrm{H}$, Kenyon C. Genes that act downstream of daf-16 to influence the lifespan of caenorhabditis elegans. Nature. 2003;424(6946):277-83.

56. Gao A, Smith R, Van Weeghel M, Kamble R, Houtkooper R. Identification of key pathways and metabolic fingerprints of longevity in c. elegans. bioRxiv; 2017.

57. Kwon G, Lee J, Koh J-H, Lim Y-H. Lifespan extension of caenorhabditis elegans by butyricicoccus pullicaecorum and megasphaera elsdenii with probiotic potential. Curr Microbiol. 2018;75(5):557-64.

58. Uno M, Honjoh S, Matsuda M, Hoshikawa H, Kishimoto S, Yamamoto T, Ebisuya M, Yamamoto T, Matsumoto K, Nishida E. A fasting-responsive signaling pathway that extends life span in c. elegans. Cell Rep. 2013;3(1): 79-91.

59. Iwasa H, Yu S, Xue J, Driscoll M. Novel egf pathway regulators modulate c. elegans healthspan and lifespan via egf receptor, plc- $\gamma$, and ip3r activation. Aging Cell. 2010;9(4):490-505.

60. Meng F, Li J, Rao Y, Wang W, Fu Y. Gengnianchun extends the lifespan of caenorhabditis elegans via the insulin/igf-1 signalling pathway. Oxidative Med Cell Longev. 2018;2018:10.

61. Sasagawa Y, Urano T, Kohara Y, Takahashi H, Higashitani A. Caenorhabditis elegans rbx 1 is essential for meiosis, mitotic chromosomal condensation and segregation, and cytokinesis. Genes to Cells. 2003;8(11):857-72.

62. Pujol C, Bratic-Hench I, Sumakovic M, Hench J, Mourier A, Baumann L, Pavlenko V, Trifunovic A. Succinate dehydrogenase upregulation destabilize complex $i$ and limits the lifespan of gas-1 mutant. PloS one. 2013;8(3):59493.

63. He K, Zhou T, Shao J, Ren X, Zhao Z, Liu D. Dynamic regulation of genetic pathways and targets during aging in caenorhabditis elegans. Aging (Albany NY). 2014;6(3):215-30.

64. Wang MC, Oakley HD, Carr CE, Sowa JN, Ruvkun G. Gene pathways that delay caenorhabditis elegans reproductive senescence. PLOS Genet. 2014;10(12):1004752. 